\title{
Effect of molecular structure on wetting behavior of water + amphiphile mixtures: A density functional approach
}

\author{
Ming-Chih Yeh, Chia-Ming Chen, and Li-Jen Chen ${ }^{\text {a) }}$ \\ Department of Chemical Engineering, National Taiwan University, Taipei 10617, Taiwan
}

(Received 30 May 2007; accepted 13 December 2007; published online 31 January 2008)

\begin{abstract}
A density functional approach is applied to investigate the effect of molecular structure on wetting behavior of water+amphiphile mixtures. The interaction-site model is employed to describe isomeric amphiphile structures. The hydrogen bonding between water and amphiphile is mimicked by energy enhancement according to specific molecular orientation. The calculations show that these systems exhibit Cahn-type criticality-related wetting transitions and pronounced adsorption behavior difference between isomeric systems. Excellent qualitative agreements with experiments are achieved. () 2008 American Institute of Physics. [DOI: 10.1063/1.2832323]
\end{abstract}

\section{INTRODUCTION}

Research on the wetting behavior of liquid-liquid-liquid and vapor/gas-liquid-liquid equilibrium systems are very important because of not only theoretical interests, but also many industrial applications such as detergent, cosmetics, insecticide, pharmacy, and tertiary oil recovery. Therefore, the wetting behavior of binary water+amphiphile mixtures and ternary water+oil+amphiphile mixtures have been widely studied both experimentally and theoretically. ${ }^{1}$

In the past two decades, the wetting behavior of ternary water+oil+amphiphile mixtures have been extensively and systematically explored. ${ }^{2}$ Considerable experimental approaches were employed to investigate the wetting behavior of the middle amphiphile-rich phase at the oil-water interface. Diverse wetting behavior can be observed by varying thermodynamic variables such as temperature, ${ }^{3}$ salinity, ${ }^{4}$ oil hydrocarbon chain length, ${ }^{5}$ and amphiphilicity of an amphiphile. ${ }^{6}$

Kahlweit and Busse ${ }^{7}$ suggested that the wetting behavior of ternary water+oil+amphiphile systems may be dominated by that of binary water+amphiphile systems based on experimental evidence. The oil-rich phase in ternary systems could only play the same role as the inert vapor/gas phase in binary systems.

The effects of temperature, ${ }^{7}$ number of hydrophilic group of an amphiphile, ${ }^{7}$ and molecular shape aniostropy ${ }^{8}$ on the mechanism of wetting transitions were verified experimentally by direct-eye observing, surface/interfacial tension measurement, and ellipsometry. In our previous studies, ${ }^{9}$ we employed pendant drop/bubble tensiometry to conclude the effects of temperature, pressure, and molecular structure on the wetting behavior of binary water+amphiphile systems.

Density functional approaches are widely used to study phase and interfacial behavior of binary and ternary systems. In early study, ${ }^{10}$ the wetting behavior of an equal-sized binary hard sphere mixture was examined by a simple version density functional theory. Recently, an off-lattice density

\footnotetext{
${ }^{a)}$ Author to whom correspondence should be addressed. Electronic mail: ljchen@ntu.edu.tw.
}

functional theory ${ }^{11}$ was developed to study ternary water +oil+amphiphile mixtures. The wetting behavior of type VI binary associating mixtures and ternary water+oil + amphiphile mixtures was successfully predicted by similar approaches. $^{12}$

In addition, the interaction-site model provides the possibility to mimic more complicated molecular structures. Adsorption of linear and branched molecules, even star polymers, at the vapor-liquid and solid-liquid interfaces was well described by the interaction-site model incorporated with density functional theory. ${ }^{13,14}$ Recently, this approach was applied to delineate the micelle, bilayer, and vesicle formation mechanisms ${ }^{15}$ and the lamellae-disorder phase boundaries ${ }^{16}$ of binary water+amphiphile mixtures.

In this work, a molecular-level study on the wetting behavior is concerned. A density functional theory is introduced to find out the influences of the hydrophobic chain length, the hydrophilic group interaction, and the geometry of an amphiphile on the wetting behavior of binary water + amphiphile mixtures.

This paper is organized as follows. The concepts of wetting behavior and wetting transition are briefly given in the next section. The free energy functionals and the calculation details are described in Sec. III. The effects of hydrophobic chain length, the hydrophilic group interaction, and the geometry of an amphiphile molecule on the wetting behavior of binary mixtures are discussed in Sec. IV. Finally, we conclude our work in Sec. V.

\section{WETTING BEHAVIOR AND WETTING TRANSITION}

Consider three fluid phases, $\alpha, \beta$, and $\gamma$, in equilibrium under gravity, and the densities of these phases are in the order $\rho_{\alpha}>\rho_{\beta}>\rho_{\gamma}$. The wetting behavior of the middle $\beta$ phase is determined according to the wetting coefficient defined as $W=\left(\sigma_{\beta \gamma}-\sigma_{\alpha \gamma}\right) / \sigma_{\alpha \beta}$. The symbol $\sigma_{i j}$ stands for interfacial tension of an interface separating $i$ and $j$ phases. Based on wetting coefficients and contact angles, wetting behavior can be categorized into three types, as illustrated in Fig. 1. 


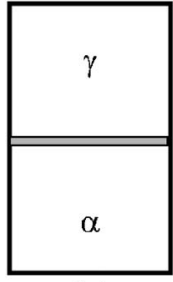

(a)

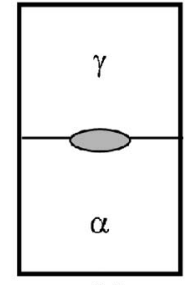

(b)

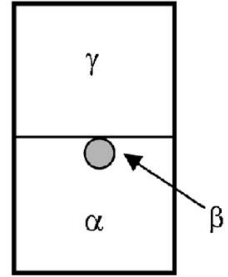

(c)
FIG. 1. Wetting behavior of $\beta$ phase at $\alpha-\gamma$ interface. (a) complete-wetting behavior, (b) partial-wetting behavior, and (c) nonwetting behavior.

Type (a): $W=-1$, a complete-wetting $\beta$ phase at the $\alpha-\gamma$ interface, as shown in Fig. 1(a). The three tensions obey Antonow's rule ${ }^{17} \sigma_{\alpha \gamma}=\sigma_{\alpha \beta}+\sigma_{\beta \gamma}$.

Type (b): $-1<W<1$, a partial-wetting $\beta$ phase at the $\alpha-\gamma$ interface, as shown in Fig. 1(b). The relationship between tensions is $\sigma_{\alpha \gamma}<\sigma_{\alpha \beta}+\sigma_{\beta \gamma}$ and $\sigma_{\beta \gamma}<\sigma_{\alpha \beta}+\sigma_{\alpha \gamma}$.

Type (c): $W=1$, a nonwetting $\beta$ phase at the $\alpha-\gamma$ interface, as shown in Fig. 1(c). Meanwhile, the $\alpha$ phase exhibits complete-wetting behavior at the $\beta$ - $\gamma$ interface. In these situations Antonow's rule applies in the form $\sigma_{\beta \gamma}=\sigma_{\alpha \beta}+\sigma_{\alpha \gamma}$.

With altering thermodynamic variables, an interfacial phase transition from partial wetting to complete wetting or vice versa may occur. Conventionally, a transition between

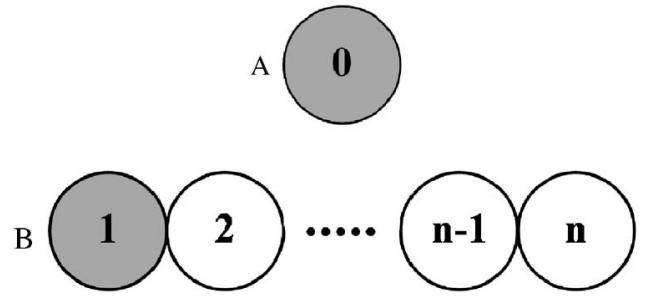

FIG. 2. The structures of model molecules A (monomer) and B (n-mer).

types (a) and (b) is called the $\beta$ phase wetting transition and the $\alpha$ phase wetting transition for a transition between types (c) and (b).

\section{MODEL}

As illustrated in Fig. 2, we consider a binary mixture consisting of spherical hard-core molecule A representing water, and multi-interaction-site molecule $\mathrm{B}$ representing amphiphile. The latter molecules are composed of $n$ tangent spheres with centers located at a fixed bond length $L$. All hard spheres of type A together with the $n$ interaction sites in molecules $\mathrm{B}$ are assumed to have the same diameter $d$. The Helmholtz free energy of this mixture may be approximated by $^{16}$

$$
\begin{aligned}
F\left[\rho_{i}(\mathbf{r})\right]= & k T \int d \mathbf{r} \rho_{0}(\mathbf{r})\left(\ln \rho_{0}(\mathbf{r})-1\right)+k T \sum_{i=1}^{n} \int d \mathbf{r} \rho_{i}(\mathbf{r}) \ln \omega_{i}(\mathbf{r})+k T \int d \mathbf{r} \Psi(\eta(\mathbf{r})) \rho_{s}(\mathbf{r})-k T \iint d \mathbf{r} d \mathbf{r}^{\prime}, \ldots, d \mathbf{r}^{n} s^{(n)} \\
& \times\left(\mathbf{r}, \mathbf{r}^{\prime}, \ldots, \mathbf{r}^{n}\right) \omega_{1}(\mathbf{r}) \omega_{2}\left(\mathbf{r}^{\prime}\right), \ldots, \omega_{n}\left(\mathbf{r}^{n}\right)+\frac{1}{2} \sum_{i, j} \iint d \mathbf{r} d \mathbf{r}^{\prime} \Phi_{i j}\left(\left|\mathbf{r}-\mathbf{r}^{\prime}\right|\right) \rho_{i}(\mathbf{r}) \rho_{j}\left(\mathbf{r}^{\prime}\right),
\end{aligned}
$$

where $k$ is the Boltzmann constant and $T$ is the absolute temperature. The free energy is a functional of local densities $\rho_{i}(\mathbf{r})$, where $i=0$ denotes the spatial density distributions of molecules $\mathrm{A}$, and $i=1-n$ corresponds to the different interaction sites of molecules B. $\omega_{i}(\mathbf{r})$ stands for the activity of the interaction site $i$ in the amphiphiles. The total density of the hard spheres and the packing fraction are given by, respectively,

$$
\begin{aligned}
& \rho_{s}(\mathbf{r})=\sum_{i=0}^{n} \rho_{i}(\mathbf{r}), \\
& \eta(\mathbf{r})=\frac{\pi d^{3}}{6} \rho_{s}(\mathbf{r}) .
\end{aligned}
$$

The first two terms on the right hand side of Eq. (1) are the ideal gas contribution to the free energy and the third term is the repulsive hard-sphere contribution which is considered in the local density approximation. For an equalsized hard-sphere mixture, the Helmholtz free energy is approximated by the Carnahan-Starling expression ${ }^{18}$

$$
\Psi(\eta)=\frac{4 \eta-3 \eta^{2}}{(1-\eta)^{2}}
$$

The fourth term on the right hand side of Eq. (1) stands for the decrease in entropy due to bonding between interaction sites $i$ to $n$ of $\mathrm{B}$ molecules. The dependence of molecular structure on free energy is taken into account by the intramolecular correlation function $s^{(n)}\left(\mathbf{r}, \mathbf{r}^{\prime}, \ldots, \mathbf{r}^{n}\right)$. For a rigid diatomic molecule of bond length $L$, the intramolecular correlation function is written as

$$
s^{(2)}\left(\mathbf{r}, \mathbf{r}^{\prime}\right)=s_{L}^{(2)}\left(\left|\mathbf{r}-\mathbf{r}^{\prime}\right|\right)=\frac{1}{4 \pi L^{2}} \delta\left(\left|\mathbf{r}-\mathbf{r}^{\prime}\right|-L\right),
$$

where the bond length $L$ is equal to $d$ in this study.

For a stiff linear-chain molecule composed of $n$ tangent hard spheres,

$$
\begin{aligned}
s^{(n)}\left(\mathbf{r}, \mathbf{r}^{\prime}, \ldots, \mathbf{r}^{n}\right)= & s_{L}^{(2)}\left(\left|\mathbf{r}^{\prime}-\mathbf{r}\right|\right) \delta\left(\mathbf{r}^{\prime \prime}-2 \mathbf{r}^{\prime}+\mathbf{r}\right) \cdots \\
& \delta\left(\mathbf{r}^{n}-2 \mathbf{r}^{n-1}+\mathbf{r}^{n-2}\right) .
\end{aligned}
$$


The last term on the right hand side of Eq. (1) is the long-range attractive interaction contribution, which includes the inverse sixth power pair potential ${ }^{19}$

$$
\Phi_{i j}(r)=-4 \varepsilon_{i j}\left(\frac{d}{r}\right)^{6} H(r-d)
$$

where $\varepsilon_{i j}$ is the energy parameter and $H$ is the Heaviside step function. The mixing rule for the interactions between sites $i$ and $j$ of $\mathrm{B}$ molecules is defined by $\varepsilon_{i j}=\left(\varepsilon_{i i} \varepsilon_{j j}\right)^{0.5}$ and the mixing rule for the interaction between A molecules, and site $j$ of B molecules is $\varepsilon_{0 j}=k_{0 j}\left(\varepsilon_{00} \varepsilon_{j j}\right)^{0.5}$, where $k_{0 j}$ stands for the mixing parameter between sites 0 and $j$.

The equilibrium density distribution functions of the mixture are obtained by minimizing the grand potential functional

$$
\Omega_{V}\left[\left\{\rho_{i}(\mathbf{r})\right\}\right]=F\left[\left\{\rho_{i}(\mathbf{r})\right\}\right]-\sum_{i=0}^{n} \int_{V} d \mathbf{r} \rho_{i}(\mathbf{r}) \mu_{i} \quad(i=0-n),
$$

where $V$ is the system volume, and $\mu_{i}$ are the chemical potentials with respect to the densities and the auxiliary functions $\omega_{i}(\mathbf{r}){ }^{16}$

$$
\begin{aligned}
& \frac{\partial \Omega}{\partial \rho_{i}(\mathbf{r})}=0 \quad(i=0-n), \\
& \frac{\partial \Omega}{\partial \omega_{i}(\mathbf{r})}=0 \quad(i=1-n) .
\end{aligned}
$$

Substituting Eq. (8) to Eq. (9) yields

$$
\begin{aligned}
& \rho_{0}(\mathbf{r})=e^{\beta\left(\mu_{0}-U_{0}(\mathbf{r})\right)} \\
& \omega_{i}(\mathbf{r})=e^{\beta\left(\mu_{i}-U_{i}(\mathbf{r})\right)},
\end{aligned}
$$

where

$$
\begin{aligned}
U_{i}(\mathbf{r})= & k T\left(\Psi(\eta(\mathbf{r}))+\frac{\partial \Psi(\eta(\mathbf{r}))}{\partial \rho_{i}(\mathbf{r})} \rho_{s}(\mathbf{r})\right) \\
& +\sum_{j=0}^{n} \int d \mathbf{r} \Phi_{i j}\left(\left|\mathbf{r}-\mathbf{r}^{\prime}\right|\right) \rho_{j}\left(\mathbf{r}^{\prime}\right) \quad(i=0-n) .
\end{aligned}
$$

While substituting Eq. (8) to Eq. (10) yields

$$
\rho_{i}(\mathbf{r})=\omega_{i}(\mathbf{r}) \int \cdots \int\left(\prod_{j \neq i} d \mathbf{r}_{j} \omega_{j}\left(\mathbf{r}_{j}\right)\right) s^{(n)}\left(\mathbf{r}, \mathbf{r}^{\prime}, \ldots, \mathbf{r}^{n}\right) .
$$

The equilibrium densities of the inhomogeneous mixture can be obtained from Eqs. (11)-(14) by solving them in planar geometry using iterative method. ${ }^{20}$ The interfacial tension between coexisting phases is easily evaluated by

$$
\sigma=\frac{\Omega_{V}\left[\left\{\rho_{i}(\mathbf{r})\right\}\right]+P V}{A} .
$$

As shown in Eq. (1), associating effect is not taken into account by the interaction-site model. To describe the phase and wetting behavior of binary water+amphiphile mixtures, the hydrogen bonding between water and amphiphile mol- ecules may be simulated by a temperature-dependent energy parameter. Vause and Walker $^{21}$ had applied a lattice-gas model to delineate the close-loop phase behavior by assigning larger attraction between $\mathrm{A}$ and $\mathrm{B}$ molecules with certain configuration. In the same manner, a temperature-dependent energy parameter between A molecules and the association site $\mathrm{H}$ of molecules $\mathrm{B}$ is proposed:

$$
\varepsilon_{0 H}(T)=\varepsilon_{0 H}+k T \ln \left[\frac{\exp \left(\varepsilon_{\mathrm{HB}} / k T\right)+q}{\exp \left(\varepsilon_{\mathrm{HH}} / k T\right)+q}\right],
$$

where $\varepsilon_{\mathrm{HB}}$ and $\varepsilon_{\mathrm{HH}}$ stand for the hydrogen bonding energy parameters between water-amphiphile and amphiphileamphiphile, respectively, and $q$ is the orientational degeneracy of a bonded pair of molecules.

\section{RESULTS AND DISCUSSION}

In a previous study, ${ }^{12}$ it was shown that the wetting behavior of binary associating liquid mixtures under vaporliquid-liquid equilibrium was similar to that under liquidliquid equilibrium. The addition of an inert gas which is used to maintain the system pressure and provide the third phase was found not to affect the nature of the wetting behavior. Consequently, it is more convenient and time saving to consider a binary liquid mixture rather than a ternary air-liquid mixture. In this study, wetting behavior of all systems was evaluated at vapor-liquid-liquid equilibrium.

All the calculations were performed in reduced units: $T^{*}=k T / \varepsilon_{00}, \quad \mu_{i}^{*}=\mu_{i} / \varepsilon_{00}, P^{*}=P d^{3} / \varepsilon_{00}, \quad \varepsilon_{i j}{ }^{*}=\varepsilon_{i j} / \varepsilon_{00}, \quad \varepsilon_{\mathrm{HB}}{ }^{*}$ $=\varepsilon_{\mathrm{HB}} / \varepsilon_{00}$, and $\rho_{i}{ }^{*}=\rho_{i} d^{3}$. For multi-interaction-site molecules $\mathrm{B}, \mathrm{B}^{n}$ is used to represent molecule $\mathrm{B}$ composed of $n$ hard spheres, i.e., an $n$-mer.

\section{A. The effect of hydrophobic chain length on the wetting behavior}

The phase and interfacial behavior of binary water + amphiphile mixtures is examined for three different chain lengths of amphiphiles: dimer $\left(\mathrm{B}^{2}\right)$, trimer $\left(\mathrm{B}^{3}\right)$, and tetramer $\left(\mathrm{B}^{4}\right)$. For $\mathrm{B}^{n}$ molecules with different lengths, the interaction site $i=1$ represents the hydrophilic head group of amphiphiles. Thus, the attraction parameter is chosen to be $\varepsilon_{11}{ }^{*}=1.1$, relatively larger than that of other hydrophobic chain segments $\varepsilon_{22}{ }^{*}=\varepsilon_{33}{ }^{*}=\varepsilon_{44}{ }^{*}=0.76$. The hydrogen bonding strength $\varepsilon_{\mathrm{HB}}{ }^{*}=5.7$ is fixed and found to be able to delineate the reentrant solubility between water and amphiphile. Figure 3(a) shows the variation of both the upper critical end point (UCEP) and lower critical end point (LCEP) as a function of $\varepsilon_{\mathrm{HB}}{ }^{*}$ for three binary systems: $\mathrm{A}+\mathrm{B}^{2}, \mathrm{~A}+\mathrm{B}^{3}$, and $\mathrm{A}+\mathrm{B}^{4}$. The enhancement of hydrogen bonding strength $\varepsilon_{\mathrm{HB}}{ }^{*}$ would shrink the immiscibility gap between $\mathrm{A}$ and $\mathrm{B}^{n}$. Both UCEP and LCEP meet at $\varepsilon_{\mathrm{HB}}{ }^{*}=5.48,5.82$, and 6.15 for the $\mathrm{A}+\mathrm{B}^{2}, \mathrm{~A}+\mathrm{B}^{3}$, and $\mathrm{A}+\mathrm{B}^{4}$ systems, respectively. For a fixed association strength, say, $\varepsilon_{\mathrm{HB}}{ }^{*}=5.7$, the effect of hydrophobic chain length of amphiphile on phase and wetting behavior is systematically further explored. The $\mathrm{B}^{2}$ molecules are completely miscible with the A molecules for $\varepsilon_{\mathrm{HB}}{ }^{*}>5.48$. While a closed-loop immiscibility gap appears for the binary 


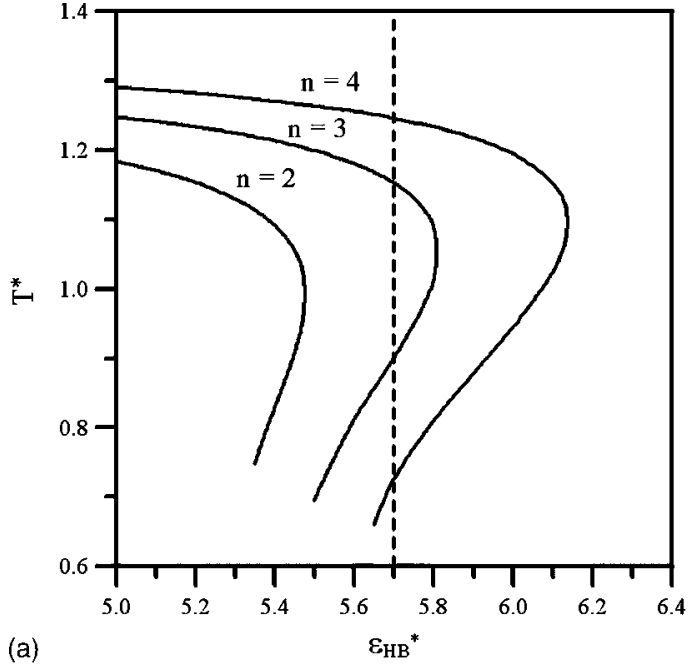

(a)

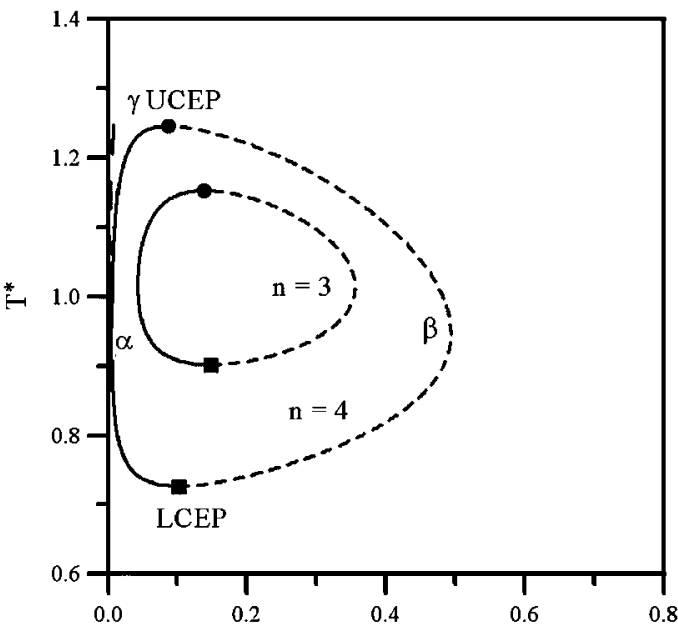

(b)

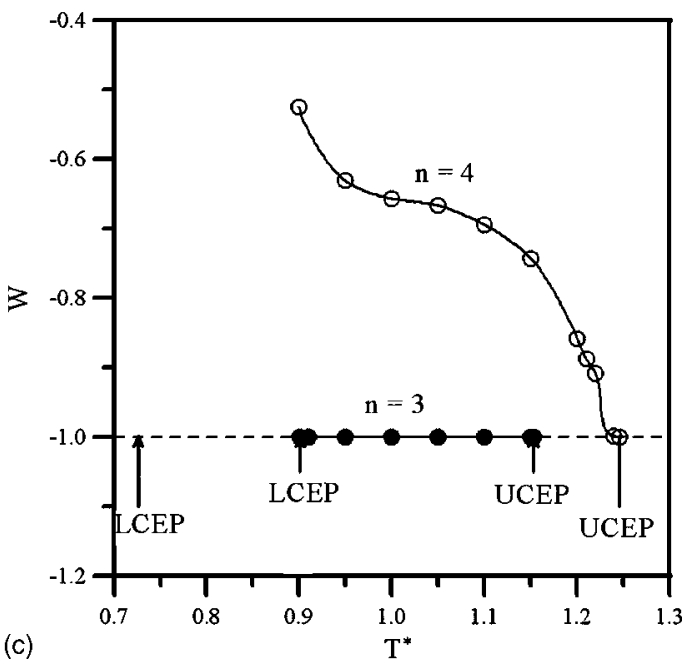

FIG. 3. (a) Effect of $\varepsilon_{\mathrm{HB}}{ }^{*}$ on the critical end points for the $\mathrm{A}+\mathrm{B}^{2}, \mathrm{~A}+\mathrm{B}^{3}$, and $\mathrm{A}+\mathrm{B}^{4}$ mixtures; (b) vapor-liquid-liquid equilibrium phase diagram for the $\mathrm{A}+\mathrm{B}^{3}$ and $\mathrm{A}+\mathrm{B}^{4}$ mixtures, UCEP $(\bullet)$ and LCEP ( $)$; and (c) wetting coefficients for the $\mathrm{A}+\mathrm{B}^{3}$ and $\mathrm{A}+\mathrm{B}^{4}$ mixtures as a function of temperature with $\varepsilon_{\mathrm{HB}}{ }^{*}=5.7$.

$\mathrm{A}+\mathrm{B}^{3}$ system, the immiscibility gap enhances along with an increase in hydrophobic chain length $n$, as illustrated in Fig. 3(b).

The wetting behavior of these binary systems is further verified according to the wetting coefficient
$W=\left(\sigma_{\beta \gamma}-\sigma_{\alpha \gamma}\right) / \sigma_{\alpha \beta}$ defined previously, where $\alpha, \beta$, and $\gamma$ stands for the A-rich, $\mathrm{B}^{n}$-rich, and vapor phases, respectively. When $n=3$, the wetting coefficient $W$ is equal to -1 throughout the whole temperature window between the upper and lower critical end points, as illustrated in Fig. 3(c). That is, the $\beta$ ( $\mathrm{B}^{3}$-rich) phase completely wets the interface separating the vapor and the A-rich phases. There always exists a significant adsorption peak with a composition of $\beta$ ( $\mathrm{B}^{3}$-rich) phase in the density profile across $\alpha-\gamma$ interface at all temperatures. As one more hydrophobic segment is added, i.e., $n$ increases from 3 to 4 , a dramatic change on the wetting behavior occurs. When the temperature is far from its UCEP for the $\mathrm{A}+\mathrm{B}^{4}$ system, the $\beta\left(\mathrm{B}^{4}\right.$-rich) phase partially wets the $\alpha-\gamma$ interface. The wetting coefficient approaches -1 until the temperature is close to its UCEP. That implies that the transition from partial wetting to complete wetting, a representative paradigm of Cahn's critical point wetting theory, ${ }^{22}$ occurs. This observation indicates that the addition of a hydrophobic segment to an amphiphile would drive the $\beta$ phase to demonstrate partial-wetting behavior.

This prediction is consistent with the recent experimental findings on water+alcohol binary systems. ${ }^{9}$ Three alcohols, butanol, pentanol, and hexanol, were used to explore the alcohol chain length effect on wetting behavior. It was found that the alcohol-rich $(\beta)$ phase has a tendency to completely wet the air-water $(\alpha-\gamma)$ interface when the alcohol chain length decreases at a fixed temperature. ${ }^{9(a)}$ In addition, a wetting transition of the alcohol-rich phase in the butanol + water system from partial wetting to complete wetting occurs when the system is driven to approach its UCEP, ${ }^{9(\mathrm{c})}$ in good agreement with our predictions shown in Fig. 3(c).

Note that when $T^{*}<0.9$ in the $\mathrm{A}+\mathrm{B}^{4}$ system, the $\beta$ phase is no longer isotropic and becomes lamellar phase, as shown in Fig. 4. In Fig. 4(a), the four distinct adsorption peaks designate ordered arrangement of $\mathrm{B}^{4}$ molecules at the $\alpha-\gamma$ interface and the orientation of hydrophilic groups (site 1 ) orientated toward the more hydrophilic $\alpha$ phase. At the $\beta-\gamma$ and $\alpha-\beta$ interfaces, it is obvious that on the $\beta$ phase side, the periodic oscillations of density profiles are formed, as shown in Figs. 4(b) and 4(c). The $\beta$ phase consists of $\mathrm{B}^{4}-\mathrm{A}-\mathrm{B}^{4}-\mathrm{A}, \ldots$ repeating multilayer structure, a signature of lamellar phase. The interfacial tensions $\sigma_{\beta \gamma}$ and $\sigma_{\alpha \beta}$ also drop dramatically and become negative before the system approaches its lower critical end point. It was pointed out that the negative interfacial tensions reflect the instability of interfaces. ${ }^{15}$ Thus, a phase transition from disorder to lamellar may occur before an interfacial wetting transition takes place. It should be noted that the lamellar phase only appears in the $\mathrm{A}+\mathrm{B}^{4}$ system at low temperatures. There exists no lamellar phase in the $\mathrm{A}+\mathrm{B}^{2}$ and $\mathrm{A}+\mathrm{B}^{3}$ systems.

\section{B. The effect of isomeric structure of amphiphile molecules on the wetting behavior}

In this section, $\mathrm{B}^{4}$ molecules with different parameter assignments and molecular structures are applied to explore the wetting behavior in the $\mathrm{A}+\mathrm{B}^{4}$ system. Three types of isomeric molecules, normal $n-\mathrm{B}^{4}$, secondary $s-\mathrm{B}^{4}$, and tertiary $t-\mathrm{B}^{4}$, are modeled, as illustrated in Fig. 5. The filled 

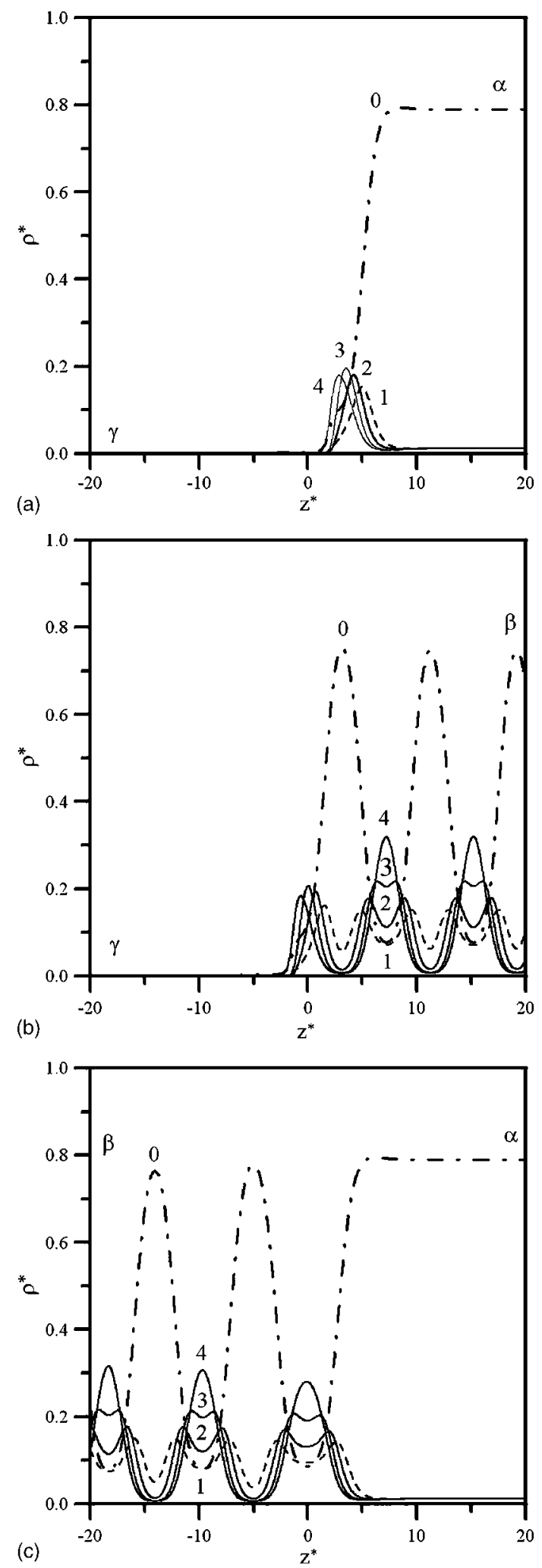

FIG. 4. Density profiles across the (a) $\alpha-\gamma$, (b) $\beta-\gamma$, and (c) $\alpha-\beta$ interfaces for $\mathrm{A}+\mathrm{B}^{4}$ mixtures with $\varepsilon_{\mathrm{HB}}{ }^{*}=5.7$ at $T^{*}=0.80$.

hard spheres stand for hydrophilic groups $\left(\varepsilon_{i i}{ }^{*}=1.1\right)$ and the open ones are the hydrophobic segments $\left(\varepsilon_{i i}{ }^{*}=0.76\right)$. The intramolecular correlation function for $t$ - $\mathrm{B}^{4}$ is considered by

$s^{(4)}\left(\mathbf{r}, \mathbf{r}^{\prime}, \mathbf{r}^{\prime \prime}, \mathbf{r}^{\prime \prime \prime}\right)=s_{L}^{(2)}\left(\left|\mathbf{r}^{\prime}-\mathbf{r}\right|\right) s_{L}^{(2)}\left(\left|\mathbf{r}^{\prime \prime}-\mathbf{r}\right|\right) s_{L}^{(2)}\left(\left|\mathbf{r}^{\prime \prime \prime}-\mathbf{r}\right|\right)$.

It should be pointed out that these three systems have
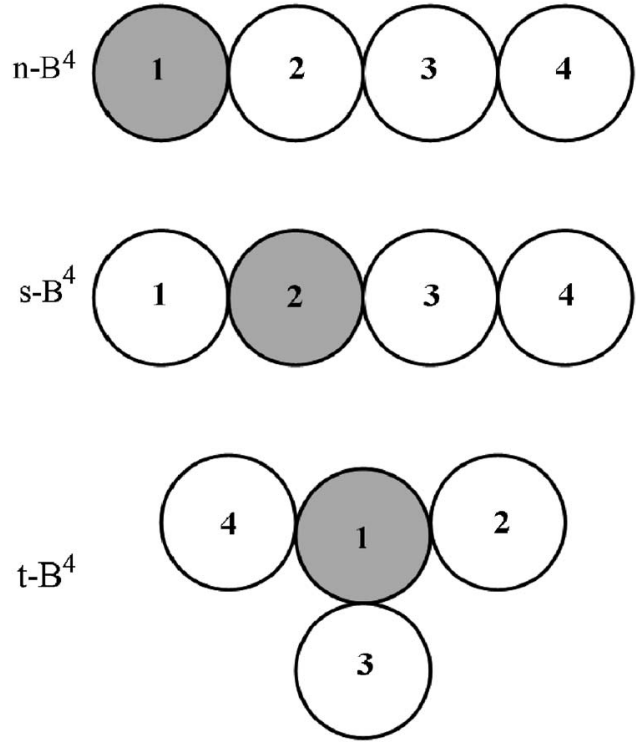

FIG. 5. Isomeric structures of $\mathrm{B}$ molecules to mimic the normal tetramer $\left(n-\mathrm{B}^{4}\right)$, secondary tetramer $\left(s-\mathrm{B}^{4}\right)$, and tertiary tetramer $\left(t-\mathrm{B}^{4}\right)$.

identical phase behavior since the isomeric $\mathrm{B}^{4}$ molecules cannot be distinguished from one another by their attractive energy parameters. Consequently, the differences of wetting behavior among these three systems can only possibly result from the isomeric structure of $\mathrm{B}^{4}$ molecules due to their different hydrophobicities. An appropriate hydrogen bonding strength $\varepsilon_{\mathrm{HB}}=5.9$ is chosen here.

Figure 6 illustrates the wetting coefficients of three A + isomeric $\mathrm{B}^{4}$ systems. Similar to the case of $\varepsilon_{\mathrm{HB}}{ }^{*}=5.7$, the $\mathrm{A}+n-\mathrm{B}^{4}$ system again exhibits an interfacial phase transition from partial wetting to complete wetting at a temperature close to its UCEP. When $T^{*}<0.95$, the $\beta$ phase becomes lamellar again. There exists no lamellar phase in the other two systems: $\mathrm{A}+s-\mathrm{B}^{4}$ and $\mathrm{A}+t-\mathrm{B}^{4}$.

The only difference between $s$ - $\mathrm{B}^{4}$ and $n$ - $\mathrm{B}^{4}$ molecules is the position of the hydrophilic groups in tetramers. As tem-

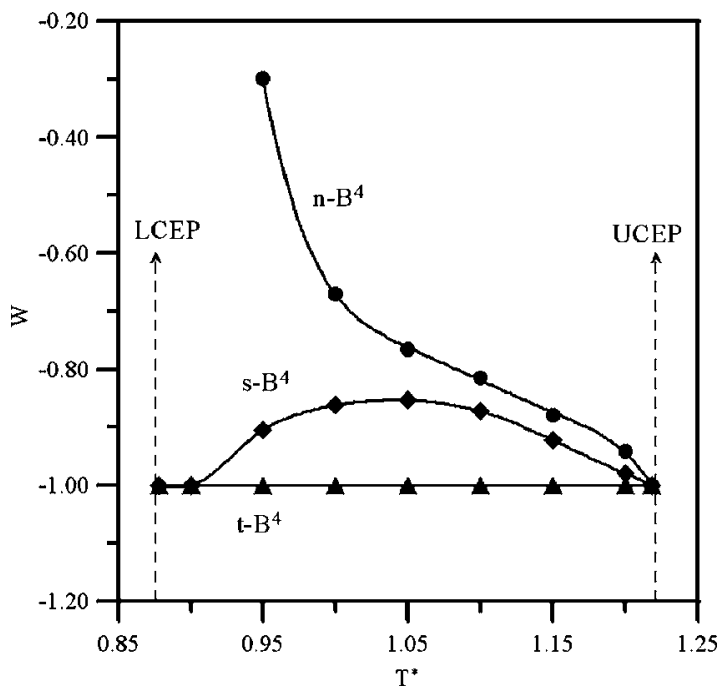

FIG. 6. Effect of isomeric structures on wetting coefficients for the A $+n-\mathrm{B}^{4}, \mathrm{~A}+s-\mathrm{B}^{4}$, and $\mathrm{A}+t-\mathrm{B}^{4}$ systems with $\varepsilon_{\mathrm{HB}}{ }^{*}=5.9$ as a function of temperature. 
peratures near the UCEP, a wetting transition from partial wetting to complete wetting occurs for the $\mathrm{A}+s-\mathrm{B}^{4}$ system. According to the temperature dependence of wetting coefficient shown in Fig. 6, when the temperature is decreased, the wetting coefficient $\mathrm{W}$ first increases to reach a maximum point at $T^{*}=1.05$, and then decreases to the value of -1 at $T^{*}=0.90$. Another wetting transition from partial wetting to complete wetting occurs. That is, the $\mathrm{A}+s-\mathrm{B}^{4}$ system exhibits a sequential wetting transition: Complete wetting $\rightarrow$ partial wetting $\rightarrow$ complete wetting, along with increasing temperature. This is the so-called re-entrant wetting behavior. $^{23}$

The wetting coefficient of $\mathrm{A}+t-\mathrm{B}^{4}$ system maintains -1 over the whole temperature range between the UCEP and the LCEP. That is, the $\beta$ ( $t-\mathrm{B}^{4}$-rich) phase always completely wets the $\alpha-\gamma$ interface in the $\mathrm{A}+t-\mathrm{B}^{4}$ system.

The diversified wetting behavior for the three isomeric systems can be further realized by density profiles across the $\alpha-\gamma$ interface. At low temperatures, e.g., $T^{*}=0.95$, the pronounced adsorption of $\mathrm{B}^{4}$ molecules exists for all the three isomeric systems, as shown in Fig. 7. The apparent four density profiles of each interaction site of $n$ - $\mathrm{B}^{4}$ molecules can be easily distinguished from one another, as shown in Fig. 7(a). In addition, the interaction site 1 (the hydrophilic head group) is orientated toward the $\alpha$ (hydrophilic) phase and all the other interaction sites are lined up in sequence 2,3 , and 4 right adjacent to the site 1 . That indicates that the $n$ - $\mathrm{B}^{4}$ molecules arrange in order across the $\alpha-\gamma$ interface. When the system switches from $\mathrm{A}+n-\mathrm{B}^{4}$ to $\mathrm{A}+s-\mathrm{B}^{4}$, the alignment of $s$ - $\mathrm{B}^{4}$ molecules across the $\alpha-\gamma$ interface does not have as much order as the $n$ - $\mathrm{B}^{4}$ molecule does, especially the density profiles of each interaction site become broader. Note that the $\beta$ phase still exhibits partial-wetting behavior for these two systems: $\mathrm{a}+n-\mathrm{B}^{4}$ and $\mathrm{A}+s-\mathrm{B}^{4}$ at $T^{*}=0.95$. For the $\mathrm{A}$ $+t$ - $\mathrm{B}^{4}$ system, the density profiles of each interaction site across the $\alpha-\gamma$ interface are almost indistinguishable from one another, as shown in Fig. 7(c), indicating the rather random adsorption behavior of $t-\mathrm{B}^{4}$ molecules at the $\alpha$ - $\gamma$ interface. It should be pointed out that the spatial distribution of A molecules is quite pronounced. The well-developed shoulder of the density profile of A molecules across the $\alpha-\gamma$ interface with the composition of $\beta$ phase emerges in the $\mathrm{A}+t-\mathrm{B}^{4}$ system, as shown in Fig. 7(c). That is, the typical evidence for the existence of a wetting layer of $\beta$ phase at the $\alpha-\gamma$ interface.

At high temperatures, e.g., $T^{*}=1.20$, all three isomeric systems exhibit complete- or near complete-wetting behavior. The density profiles for four interaction sites of $\mathrm{B}^{4}$ molecules at the $\alpha-\gamma$ interface are almost indistinguishable for these systems, as shown in Fig. 8. The ordered adsorption behavior, dominated by hydrogen bonding and the structure effects, observed at low temperatures is weakening by the entropic mixing.

This prediction of the wetting behavior in the water + isomeric $\mathrm{B}^{4}$ systems is consistent with the recent experimental findings on water $+\mathrm{C}_{i} \mathrm{E}_{j}$ binary systems, ${ }^{9}$ where the symbol $\mathrm{C}_{i} \mathrm{E}_{j}$ stands for the abbreviation of a nonionic polyoxyethylene alcohol $\mathrm{C}_{i} \mathrm{H}_{2 i+1}\left(\mathrm{OCH}_{2} \mathrm{CH}_{2}\right)_{j} \mathrm{OH}$. Three alcohols, $n-\mathrm{C}_{5} \mathrm{E}_{0}, s-\mathrm{C}_{5} \mathrm{E}_{0}$, and $t-\mathrm{C}_{5} \mathrm{E}_{0}$, were used to explore the
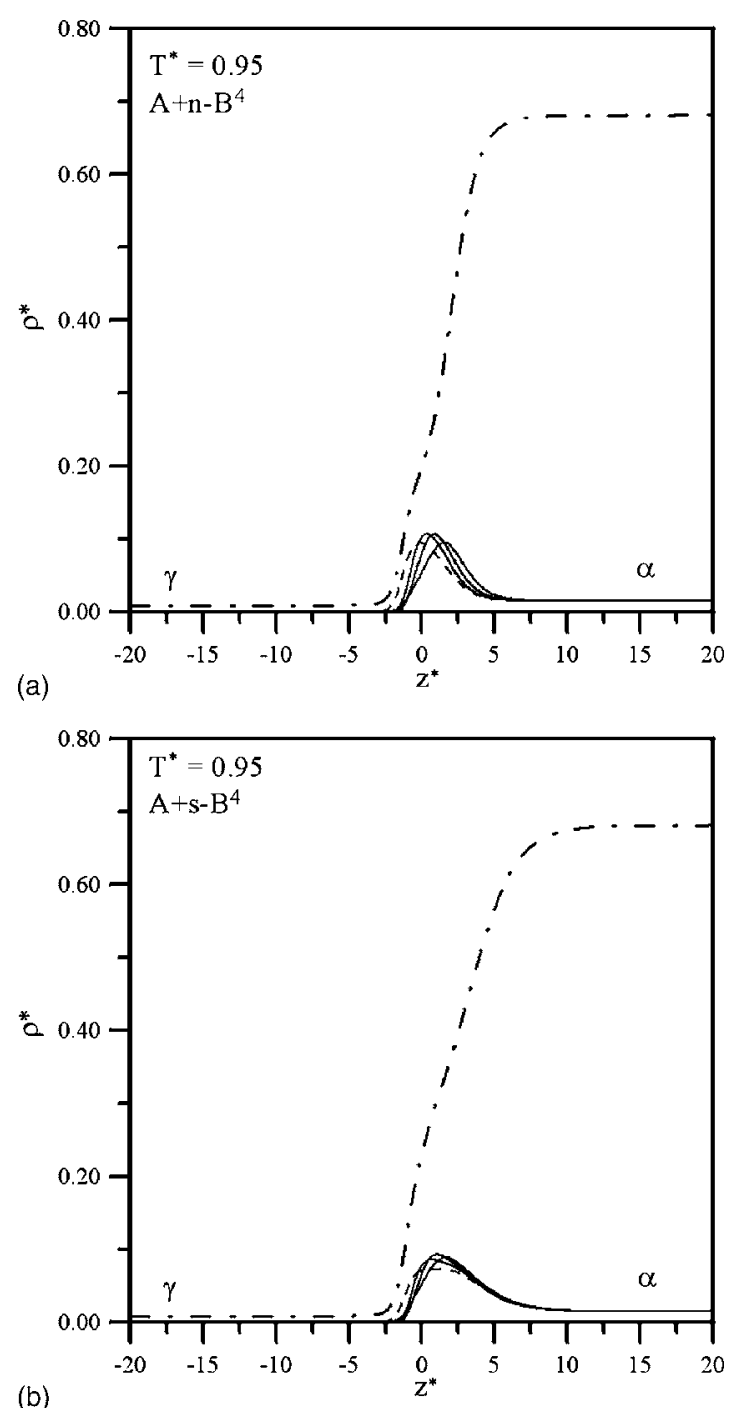

(b)

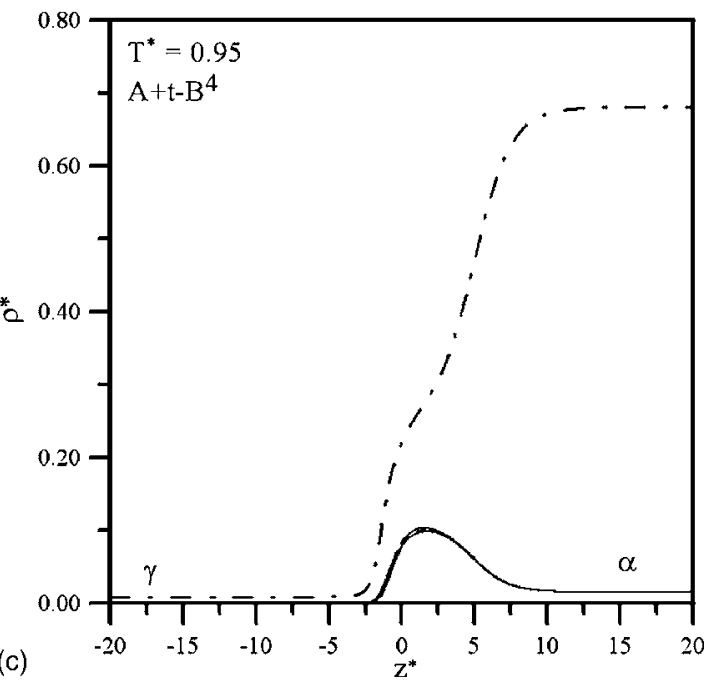

FIG. 7. Density profiles across the $\alpha-\gamma$ interface for the (a) $\mathrm{A}+n-\mathrm{B}^{4}$, (b) $\mathrm{A}+s-\mathrm{B}^{4}$, and (c) $\mathrm{A}+t-\mathrm{B}^{4}$ systems with $\varepsilon_{\mathrm{HB}}{ }^{*}=5.9$ at $T^{*}=0.95$.

isomeric structure effect on wetting behavior. It was found that the wetting coefficient for the water $+t-\mathrm{C}_{5} \mathrm{E}_{0}$ system remains equal to -1 over the experimental temperature ranging from 10 to $40{ }^{\circ} \mathrm{C} .{ }^{9(a)}$ It was found that the alcohol-rich $(\beta)$ phase has a tendency to completely wet the air-water $(\alpha-\gamma)$ 


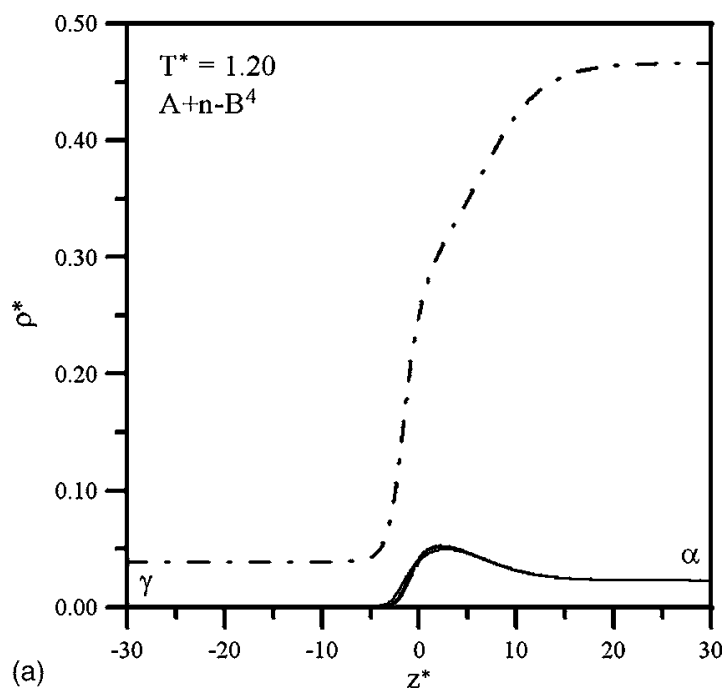

(a)

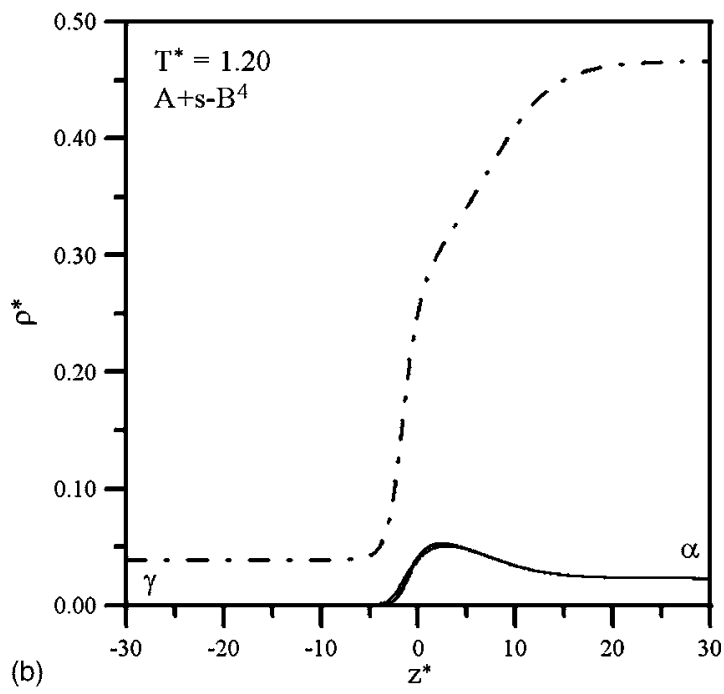

(b)

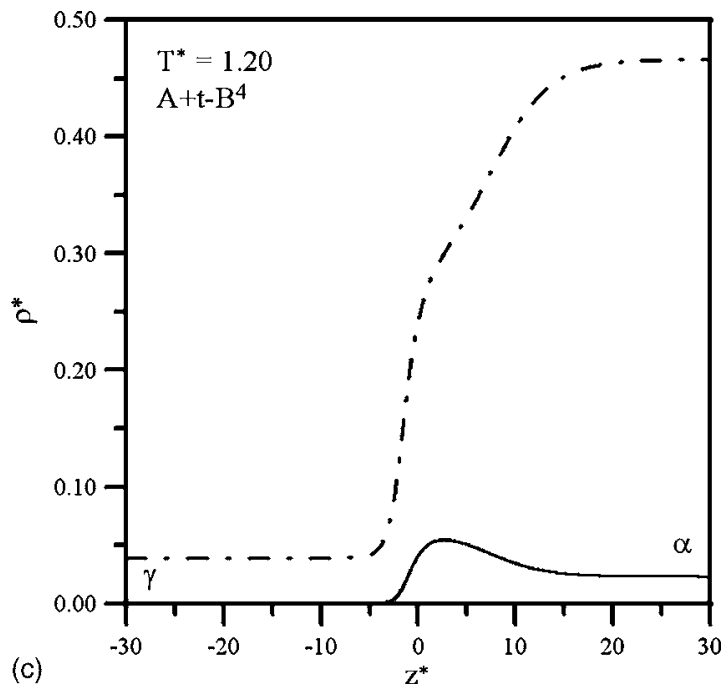

FIG. 8. Density profiles across the $\alpha-\gamma$ interface for A+ (a) $n-\mathrm{B}^{4}$, (b) $s-\mathrm{B}^{4}$ and (c) $t$ - $\mathrm{B}^{4}$ mixtures with $\varepsilon_{\mathrm{HB}}{ }^{*}=5.9$ at $T^{*}=1.20$.

interface when the effective chain length of alcohol decreases at a fixed temperature. ${ }^{9(a)}$ The effective chain length for the systems of interest is in the order $n-\mathrm{C}_{5} \mathrm{E}_{0}>s-\mathrm{C}_{5} \mathrm{E}_{0}$ $>t-\mathrm{C}_{5} \mathrm{E}_{0}$. Our prediction shown in Fig. 6 is in excellent agreement with these experimental results. However, there

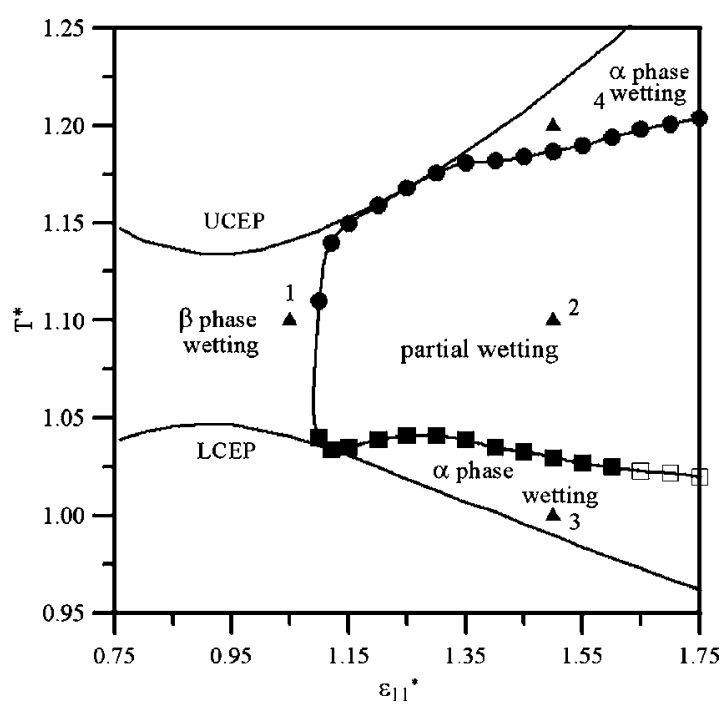

FIG. 9. Variation of UCEP, LCEP, and wetting behavior for the $\mathrm{A}+n-\mathrm{B}^{4}$ system as a function $\varepsilon_{11}{ }^{*}$. Circle and square symbols stand for wetting transition temperature near UCEP and LCEP, respectively. Filled symbol stands for the second order transition and open symbol for the first order transition.

are no experimental data to verify wetting behavior for the water $+s-\mathrm{C}_{5} \mathrm{E}_{0}$ and water $+n-\mathrm{C}_{5} \mathrm{E}_{0}$ systems driven to approach its LCEP.

\section{Effect of the hydrophilic interaction between amphiphiles on the wetting behavior}

Effect of attraction parameter of hydrophilic groups, $\varepsilon_{11}{ }^{*}$, on wetting behavior is essential. As mentioned above, the lamellar phase always appears at low temperatures, e.g., $T^{*}<0.9$ for the $\mathrm{A}+n-\mathrm{B}^{4}$ system of $\varepsilon_{\mathrm{HB}}{ }^{*}=5.70$. In order to avoid the system to run into the lamellar phase, the $\mathrm{A}$ $+n$ - $\mathrm{B}^{4}$ system with stronger hydrogen bonding strength $\varepsilon_{\mathrm{HB}}{ }^{*}=6.11$ is chosen since larger $\varepsilon_{\mathrm{HB}}{ }^{*}$ would induce higher LCEP. The variation of UCEP and LCEP as a function of $\varepsilon_{11}{ }^{*}$ is illustrated in Fig. 9. The system exhibits vapor-liquidliquid-three-phase coexistence over the temperature window between the UCEP and LCEP. Note that the LCEP is always higher than 0.96 over the whole range of $0.76<\varepsilon_{11}{ }^{*}<1.75$, as shown in Fig. 9. It is found that there is no lamellar phase in the three-phase coexistence region of the system with $\varepsilon_{\mathrm{HB}}{ }^{*}=6.11$.

The wetting behavior of $\beta$ phase in this three-phase coexistence region can be classified into four regions: (a) Complete wetting of $\beta$ phase, (b) partial wetting, (c) complete wetting of $\alpha$ phase near the LCEP, and (d) complete wetting of $\alpha$ phase near the UCEP.

As the interaction parameter between hydrophilic groups is equal to or slightly larger than that between hydrophobic chains, $\varepsilon_{11}{ }^{*}=0.76-1.08$, the $\beta\left(\mathrm{B}^{4}\right.$-rich $)$ phase completely wets the interface separating the vapor $(\gamma)$ - and A-rich $(\alpha)$ phases in the entire immiscibility gap. The typical density profiles of each interaction site in this regime with $\varepsilon_{11}$ $=1.05$ at $T^{*}=1.10$ (triangle 1 in Fig. 9) are shown in Fig. 10. An obvious wetting layer with the compositions of the $\beta$ phase propagates at vapor-A-rich interface. At $\alpha-\beta$ and $\beta$ - $\gamma$ 


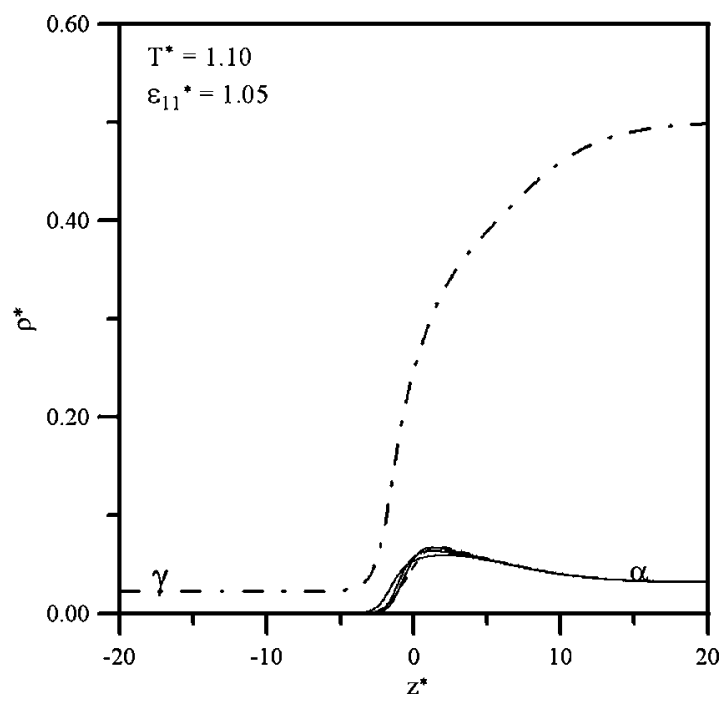

FIG. 10. Density profiles across the $\alpha-\gamma$ interface for the $\mathrm{A}+n-\mathrm{B}^{4}$ system with $\varepsilon_{11}{ }^{*}=1.05$ at $T^{*}=1.10$.

interfaces, density profiles of $\mathrm{A}$ and $\mathrm{B}^{4}$ exhibit monotonically variations across the interfaces, except that a small amount of $\mathrm{B}^{4}$ would adsorb at vapor- $\mathrm{B}^{4}$-rich interface.

When $\varepsilon_{11}{ }^{*}$ is increased up to 1.50 at constant $T^{*}=1.10$ (triangle 2 in Fig. 9), significant changes on adsorption behavior at the $\alpha-\gamma$ and $\beta-\gamma$ interfaces can be observed, as shown in Fig. 11. There is a substantial amount of A molecules adsorbed at the $\beta-\gamma$ interface indicating a premature wetting layer and an obvious thin layer of $\mathrm{B}^{4}$ molecules with a certain order alignment evolves at the $\alpha$ - $\gamma$ interface. It is also clear that the favorable adsorption of A molecules inhibits the local density of $\mathrm{B}^{4}$ molecules at the $\alpha-\gamma$ interface.

Consider a system of $\varepsilon_{11}{ }^{*}>1.08$, say, $\varepsilon_{11}{ }^{*}=1.50$ and $T^{*}=1.10$, the $\alpha$ phase always exhibits partial-wetting behavior at the $\beta-\gamma$ interface. The system can be driven to an interfacial phase transition of $\alpha$ phase from partial wetting to complete wetting by either raising or lowering temperature. In other words, when the temperature is decreased, the system exhibits a wetting transition of $\beta$ phase from partial wetting to nonwetting. Figure 12 illustrates the density profiles across the $\alpha-\gamma$ and $\beta-\gamma$ interfaces under the condition of $\varepsilon_{11}{ }^{*}=1.50$ and $T^{*}=1.00$ (triangle 3 in Fig. 9). It is obvious that interfacial enrichment is observed for both molecules A and $\mathrm{B}^{4}$ at the $\alpha-\gamma$ interface, as shown in Fig. 12(a). A welldeveloped $\alpha$ phase wetting layer adsorbs at the $\beta$ - $\gamma$ interface, as shown in Fig. 12(b). Although the $\mathrm{B}^{4}$ molecules adsorb orderly, the wetting layer mainly consists of A molecules, indicating that the A molecules dominate the wetting behavior for large $\varepsilon_{11}{ }^{*}$ systems. It is plausible that the large $\varepsilon_{11}{ }^{*}$ inhibits the adsorption of $\mathrm{B}^{4}$ molecules at the interface due to an increase in the free energy of the system. Instead, the $\mathrm{B}^{4}$ molecules adsorb orderly to form a monolayerlike structure to reduce the system free energy. This monolayerlike structure of $\mathrm{B}^{4}$ molecules can be easily observed at both the $\alpha-\gamma$ and $\beta-\gamma$ interfaces, as shown in Fig. 12. Similar adsorption behavior of both vapor-liquid interfaces was demonstrated by Kahlweit and Busse $^{7}$ with many water+amphiphile systems.

For the regime in the upper right corner of Fig. 9, the $\alpha$
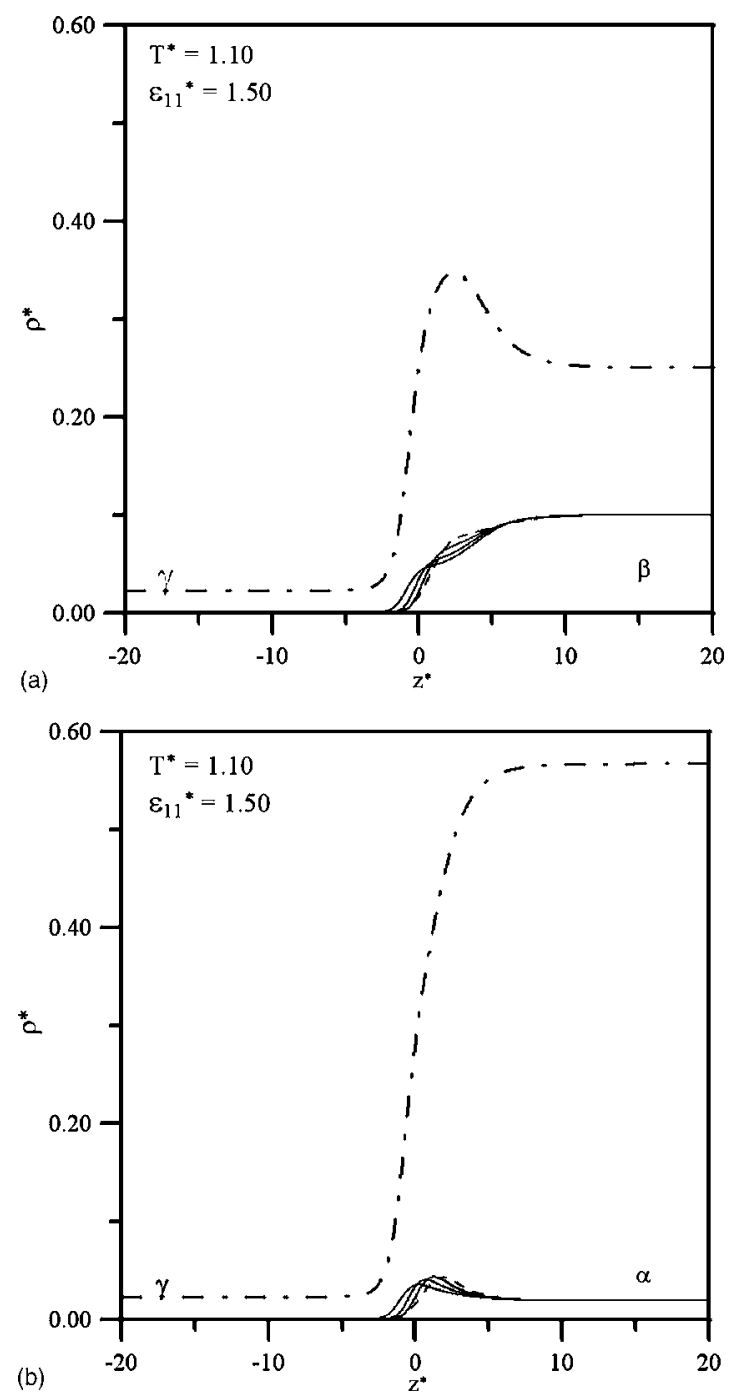

FIG. 11. Density profiles across the (a) $\alpha-\gamma$ and (b) $\beta$ - $\gamma$ interfaces for the $\mathrm{A}+n-\mathrm{B}^{4}$ system with $\varepsilon_{11}{ }^{*}=1.50$ at $T^{*}=1.10$.

phase also exhibits complete-wetting behavior at the $\beta$ - $\gamma$ interface. Figure 13 illustrates the density profiles across the $\alpha-\gamma$ and $\beta-\gamma$ interfaces under the condition of $\varepsilon_{11}{ }^{*}=1.50$ and $T^{*}=1.20$ (triangle 4 in Fig. 9). Unlike the lower temperature cases, there exists no monolayerlike structure of $\mathrm{B}^{4}$ molecules at the $\alpha-\gamma$ and $\beta-\gamma$ interfaces. In addition, the amphiphilic $\mathrm{B}^{4}$ molecules show no adsorption advantage at the $\alpha-\gamma$ and $\beta-\gamma$ interfaces.

It is interesting to note that there exists a sequence of wetting transitions as a function of temperature under the condition of $\varepsilon_{11}{ }^{*}=1.15$. That is, the $\beta$ phase exhibits a sequence of wetting transitions: Nonwetting $\rightarrow$ partial wetting $\rightarrow$ complete wetting at the $\alpha-\gamma$ interface while the temperature is increased, consistent with the wetting behavior observed in the water $+n-\mathrm{C}_{4} \mathrm{E}_{1}$ and water $+i-\mathrm{C}_{4} \mathrm{E}_{1}$ systems. ${ }^{7,9}$

In this study, the order of wetting transitions is also determined by strictly following the method of Tarazona and Evans. ${ }^{20(b)}$ A wetting transition is said to be first order if the temperature dependence of wetting film thickness exhibits a discontinuity at the wetting transition temperature. On the other hand, if the wetting film thickness grows gradually and diverges at the wetting transition temperature, the wetting 

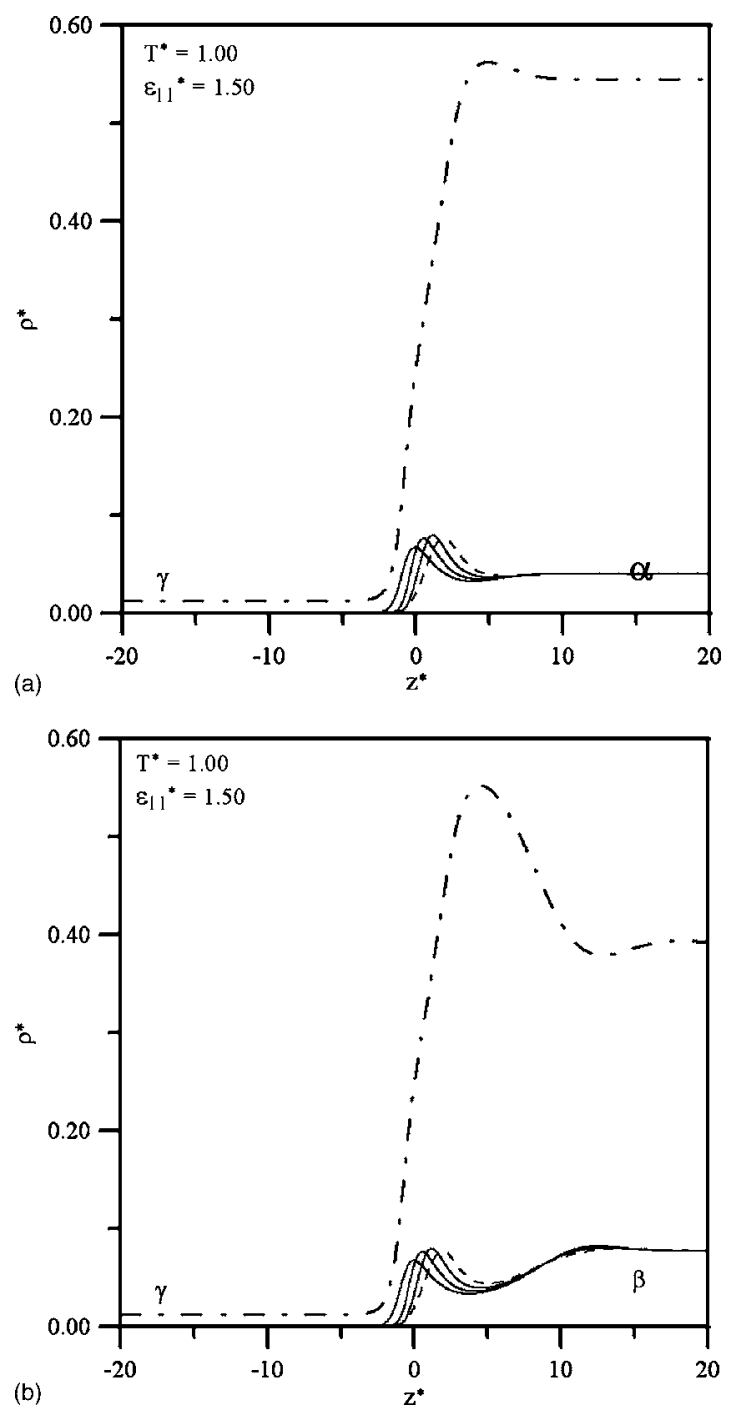

FIG. 12. Density profiles across the (a) $\alpha-\gamma$ and (b) $\beta$ - $\gamma$ interfaces for the A $+n$ - $\mathrm{B}^{4}$ system with $\varepsilon_{11}{ }^{*}=1.50$ at $T^{*}=1.00$.

transition is identified as second order. The variation of the order of wetting transitions as a function of $\varepsilon_{11}{ }^{*}$ is also shown in Fig. 9. All the wetting transitions near the UCEP are second order, indicated by filled circle symbol in Fig. 9. The wetting transitions near the LCEP are also second order for $\varepsilon_{11}{ }^{*}<1.625$ (indicated by filled square symbol in Fig. 9) and first order for $\varepsilon_{11}{ }^{*}>1.625$ (indicated by open square symbol).

\section{CONCLUSION}

The interaction-site model associated with the density functional theory is applied to explore the molecular structure effect on wetting behaviors at the air-liquid interface of water+amphiphile mixtures. Three different effects of molecular structure are examined. (1) The effect of hydrophobic chain length of amphiphile molecules on the phase and wetting behaviors are delineated by using three different chain lengths of amphiphile molecules. An increase in the hydrophobic chain length would enhance the immiscibility gap and drive the system from complete wetting to partial wetting under the condition of fixed interaction parameters. It is
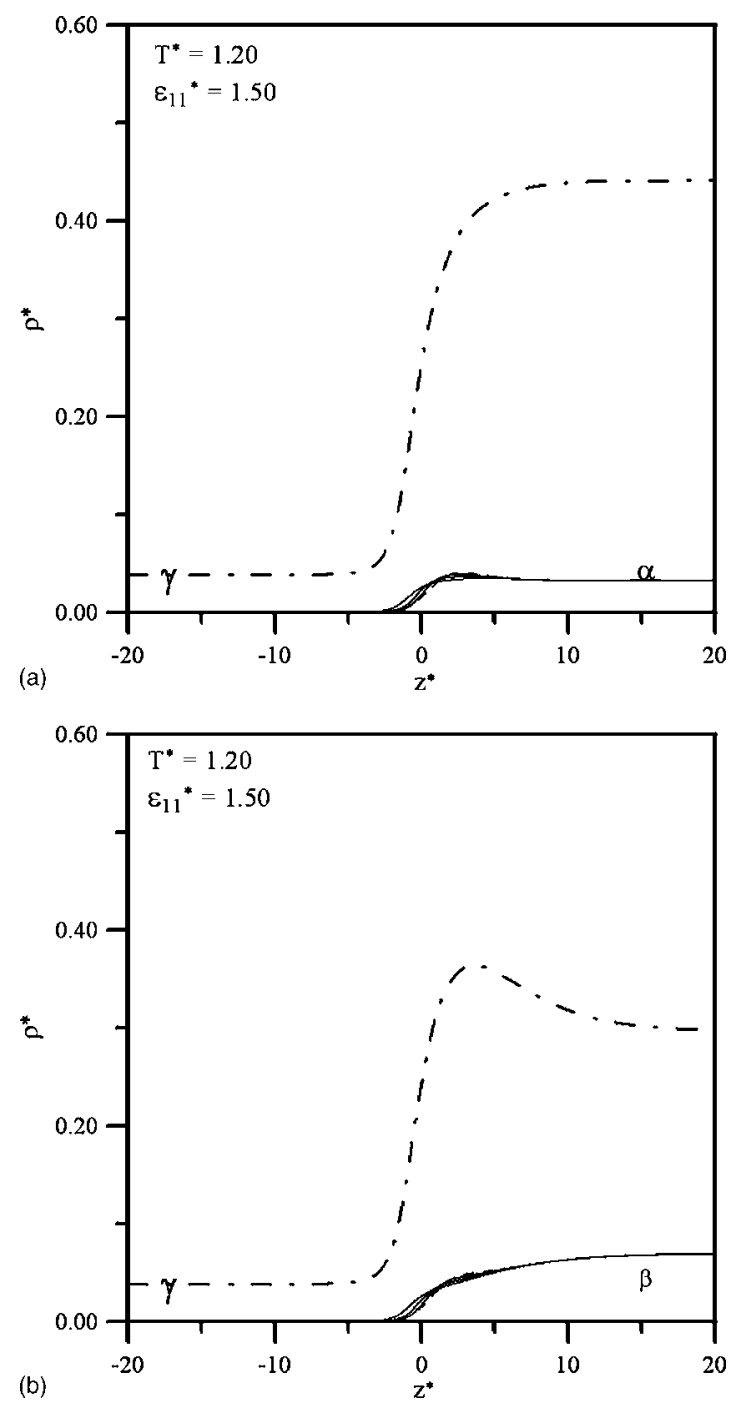

FIG. 13. Density profiles across the (a) $\alpha-\gamma$ and (b) $\beta$ - $\gamma$ interfaces for the $\mathrm{A}+n-\mathrm{B}^{4}$ system with $\varepsilon_{11}{ }^{*}=1.50$ at $T^{*}=1.20$.

interesting to observe the lamellar phase in the binary system with long hydrophobic chain amphiphile, but not for short ones, at low temperatures, especially close to its lower critical end point. (2) An amphiphile molecule can be described as a linear $\left(n-\mathrm{B}^{4}\right)$, branched $\left(s-\mathrm{B}^{4}\right)$, or spherical $\left(t-\mathrm{B}^{4}\right)$ multisegment molecule. The $\beta\left(t-\mathrm{B}^{4}\right.$-rich $)$ phase always completely wets the $\alpha-\gamma$ interface in the $\mathrm{A}+t-\mathrm{B}^{4}$ system. For the other two systems, $\mathrm{A}+n-\mathrm{B}^{4}$ and $\mathrm{A}+s-\mathrm{B}^{4}$, the $\beta$ phase exhibits partial-wetting behavior and the wetting transition from partial to complete wetting occurs when the system is driven to approach its critical end point, consistent with the prediction of Cahn's critical wetting theory. (3) For a fixed hydrophobic chain length of amphiphile, the global phase and wetting behaviors are delineated in Fig. 9 by scanning the attraction parameter of hydrophilic groups $\varepsilon_{11}{ }^{*}$. In general, the $\beta$ phase always exhibits complete-wetting behavior when $\varepsilon_{11}{ }^{*}<1.08$. On the other hand, while $\varepsilon_{11}{ }^{*}$ is large, the $\alpha$ phase exhibits a sequence of wetting transitions: complete wetting $\rightarrow$ partial wetting $\rightarrow$ complete wetting at the interface separating the $\beta$ and $\gamma$ phases along with increasing temperature. The order of wetting transitions is also discussed. Excellent agreements with recent experimental results ${ }^{7,9}$ are 
demonstrated. It should be noted that the $\beta$ phase exhibits a sequence of wetting transitions: Nonwetting $\rightarrow$ partial wetting $\rightarrow$ complete wetting at the $\alpha-\gamma$ interface while the temperature is increased under the condition of $\varepsilon_{11}{ }^{*}=1.15$ in Fig. 9. This observation is consistent with the wetting behavior in the water $+C_{i} E_{j}$ system. $^{3-5,7,9}$ The lamellar phase is also observed in certain temperature region. Currently, we are in the process of exploring the phase diagram for water + amphiphile binary mixtures by using the interaction-site model. That is, we would like to further determine the phase boundary of the lamellar phase.

${ }^{1}$ S. Dietrich, in Phase Transitions and Critical Phenomena, edited by C. Domb and J. L. Lebowitz (Academic, London, 1988), Vol. 12, p. 1; G. Gompper and M. Schick, in Phase Transitions and Critical Phenomena, edited by C. Domb and J. L. Lebowitz (Academic, New York, 1994), Vol. 16; D. Bonn and D. Ross, Rep. Prog. Phys. 64, 1085 (2001).

${ }^{2}$ M. Kahlweit, Annu. Rep. Prog. Chem., Sect. C: Phys. Chem. 95, 89 (1999); L.-J. Chen, in Encyclopedia of Surface and Colloid Science, edited by A. Hubbard (Dekker, New York, 2002), p. 5630.

${ }^{3}$ L.-J. Chen and W.-J. Yan, J. Chem. Phys. 98, 4830 (1993).

${ }^{4}$ L.-J. Chen and M.-C. Hsu, J. Chem. Phys. 97, 690 (1992).

${ }^{5}$ L.-J. Chen, C.-D. Chiu, F.-S. Shau, W.-J. Cheng, and J.-G. Wu, J. Phys.
Chem. B 106, 12782 (2002).

${ }^{6}$ M. Aratono and M. Kahlweit, J. Chem. Phys. 95, 8578 (1991).

${ }^{7}$ M. Kahlweit and G. Busse, J. Chem. Phys. 91, 1339 (1989).

${ }^{8}$ A. Hirtz, K. Bonkhoff, and G. H. Findenegg, Adv. Colloid Interface Sci. 44, 241 (1993)

${ }^{9}$ M.-C. Yeh, P.-C. Lin, and L.-J. Chen, J. Phys. Chem. B 108, 9955 (2004); C.-K. Wu and L.-J. Chen, J. Chem. Phys. 123, 084506 (2005); Langmuir 21, 6883 (2005); J. Phys. Chem. B 110, 11907 (2006).

${ }^{10}$ M. M. Telo da Gama and R. Evans, Mol. Phys. 48, 229 (1983).

${ }^{11}$ V. Talanquer and D. W. Oxtoby, Faraday Discuss. 112, 91 (1999).

${ }^{12}$ M.-C. Yeh and L.-J. Chen, J. Chem. Phys. 118, 8331 (2003); C.-M. Chen, M.-C. Yeh, and L.-J. Chen, J. Phys. Chem. B 110, 3294 (2006).

${ }^{13}$ T. A. Cherepanova and A. V. Stekolnikov, Mol. Phys. 82, 125 (1994).

${ }^{14}$ T. A. Cherepanova and A. V. Stekolnikov, Mol. Phys. 83, 1065 (1994).

${ }^{15}$ V. Talanquer and D. W. Oxtoby, J. Chem. Phys. 113, 7013 (2000).

${ }^{16}$ I. Napari, A. Laaksonen, and R. Strey, J. Chem. Phys. 113, 4476 (2000).

${ }^{17}$ G. N. Antonow, J. Chim. Phys. Phys.-Chim. Biol. 5, 372 (1907).

${ }^{18}$ N. F. Carnahan and K. E. Starling, J. Chem. Phys. 51, 635 (1969).

${ }^{19}$ J. S. Rowlinson and F. L. Swinton, Liquid and Liquids Mixtures, 3rd ed. (Butterworth, London, 1982).

${ }^{20}$ P. Tarazona, M. M. Telo da Gama, and R. Evans, Mol. Phys. 49, 283 (1983); P. Tarazona and R. Evans, ibid. 48, 799 (1983).

${ }^{21}$ C. A. Vause and J. S. Walker, Phys. Lett. 90A 419 (1982).

${ }^{22}$ J. W. Cahn, J. Chem. Phys. 66, 3667 (1977).

${ }^{23}$ C. Pérez, P. Roquero, and V. Talanquer, J. Chem. Phys. 100, 5913 (1994). 